\title{
Hadamard Matrices, Finite Sequences, and Polynomials Defined on the Unit Circle
}

\author{
By C. H. Yang
}

\begin{abstract}
If a (*)-type Hadamard matrix of order $2 n$ (i.e. a pair $(A, B)$ of $n \times n$ circulant $(1,-1)$ matrices satisfying $\left.A A^{\prime}+B B^{\prime}=2 n I\right)$ exists and a pair of Golay complementary sequences (or equivalently, two-symbol $\delta$-code) of length $m$ exists, then a (*)-type Hadamard matrix of order $2 m n$ also exists. If a Williamson matrix of order $4 n$ (i.e. a quadruple $(W, X, Y, Z)$ of $n \times n$ symmetric circulant $(1,-1)$ matrices satisfying $\left.W^{2}+X^{2}+Y^{2}+Z^{2}=4 n I\right)$ exists and a four-symbol $\delta$-code of length $m$ exists, then a Goethals-Seidel matrix of order $4 m n$ (i.e. a quadruple $(A, B, C, D)$ of $m n \times m n$ circulant $(1,-1)$ matrices satisfying $\left.A A^{\prime}+B B^{\prime}+C C^{\prime}+D D^{\prime}=4 m n l\right)$ also exists. Other related topics are also discussed.
\end{abstract}

A sequence $\left(c_{k}\right)$ is called a $(d, e)$ sequence if each $c_{k}=d$ or $e$. A finite $(d, e)$ sequence $C_{n}=\left(c_{k}\right)_{n}=\left(c_{1}, c_{2}, \ldots, c_{n}\right)$ can be associated with a polynomial $C_{n}(z)=$ $\Sigma_{1}^{n} c_{k} z^{k-1}$, where $z=\exp (i x), x$ is a real number and $i=\sqrt{-1}$.

Definition. Two $(1,-1)$ sequences $A_{n}=\left(a_{k}\right)_{n}$ and $B_{n}=\left(b_{k}\right)_{n}$ are said to be a pair of Golay complementary sequences of length $n$ (abbreviated as GCL(n)), if their associated polynomials $A_{n}(z)$ and $B_{n}(z)$ satisfy

$$
\left|A_{n}(z)\right|^{2}+\left|B_{n}(z)\right|^{2}=2 n \text { for any complex number } z
$$

on the unit circle $K=\{z \in \mathbf{C}:|z|=1\}=\{z: z=\exp (i x), 0 \leqslant x \leqslant 2 \pi\}$, where $\mathbf{C}$ is the complex field.

Let $c(j)=\sum_{k=1}^{n-j} c_{k} c_{k+j}$ for a given sequence $\left(c_{k}\right)_{n}$. The condition (1) is also equivalent to the following Golay definition of $\operatorname{GCL}(n)$ (see [2]),

$$
a(j)+b(j)=0 \text { for } j \neq 0 \text { (i.e. } 1 \leqslant j \leqslant n-1) .
$$

The above can be proved easily by observing that $\left|C_{n}(z)\right|^{2}=C_{n}(z) C_{n}\left(z^{-1}\right)=c(0)+$ $\Sigma_{1}^{n-1} c(k)\left(z^{k}+z^{-k}\right), c(0)=\Sigma c_{k}^{2}=n$, and $z^{k}+z^{-k}=2 \cos k x$ for $z=\exp (i x)$.

Definition. Two finite $(1,-1)$ sequences $A=\left(a_{k}\right)_{n}$ and $B=\left(b_{k}\right)_{n}$ are said to be a pair of Hadamard sequences of length $n$ (abbreviated as HL(n)), if their associated polynomials $A(w)$ and $B(w)$ satisfy

$$
|A(w)|^{2}+|B(w)|^{2}=2 n \quad \text { for any } w \in K_{n},
$$

where $K_{n}=\left\{w \in \mathbf{C}: w^{n}=1\right\}$ is the set of all $n$th roots of unity. We shall omit the subscript $n$ of $C_{n}$ or $\left(c_{k}\right)_{n}$ from now on if there is no confusion. Let $c^{*}(j)=c(j)+$ $c(n-j)=\Sigma_{1}^{n} c_{k} c_{k+j}$, where the subscript $k+j$ is congruent modulo $n$. Then

Received August 30, 1976.

AMS (MOS) subject classifications (1970). Primary 05B20, 05A19, 62 K05. 
$|C(w)|^{2}=C(w) C\left(w^{-1}\right)=\Sigma_{0}^{n-1} c^{*}(k) w^{k}$, where $c^{*}(0)=n$, consequently the condition (3) is also equivalent to the following

$$
\left.a^{*}(j)+b^{*}(j)=0 \text { for } j \neq 0 \text { (i.e. } 1 \leqslant j \leqslant n / 2\right) .
$$

We note here that $c^{*}(n-j)=c^{*}(j)$. Since $K_{n} \subset K$, we obtain

LEMMA 1. If $\left(a_{k}\right)$ and $\left(b_{k}\right)$ are a pair of $\operatorname{GCL}(n)$, then they are also a pair of $\mathrm{HL}(n)$.

It should be noted that if $A=\left(a_{k}\right)$ is a $\operatorname{GCL}(n)$ then $-A=\left(-a_{k}\right)=$ $\left(-a_{1},-a_{2}, \ldots,-a_{n}\right)$ and $A^{r}=\left(a_{k}^{r}\right)=\left(a_{n-k+1}\right)=\left(a_{n}, \ldots, a_{2}, a_{1}\right)$ are also $\operatorname{GCL}(n)$. Similarly, if $A=\left(a_{k}\right)$ is an $\operatorname{HL}(n)$, then $-A, A^{r}$, and $A^{(j)}=\left(a_{k}^{(j)}\right)=\left(a_{k+j}\right)=$ $\left(a_{j+1}, \ldots, a_{n}, a_{1}, \ldots, a_{j}\right)$, for $1 \leqslant j \leqslant n-1$, are also $\operatorname{HL}(n)$. GCL( $(n)$ and $\operatorname{HL}(n)$ exist only if $n=1$ or $n$ is even (see [2], [16], [17]).

When $\left(a_{k}\right)$ and $\left(b_{k}\right)$ are a pair of $\mathrm{HL}(n)$, they can be regarded as the first row entries of $n \times n$ circulant matrices $A$ and $B$, respectively, such that

$$
M=\left(\begin{array}{cc}
A & B \\
-B^{\prime} & A^{\prime}
\end{array}\right)
$$

is an Hadamard matrix of order $2 n$, i.e. $M M^{\prime}=2 n I$, since $A A^{\prime}+B B^{\prime}=2 n I_{n}$, where ' indicates the transposed and $I$ is the identity matrix. (See [16] , [17].) Such a Hadamard matrix $M$ is said to be of (*)-type.

Definition. A quadruple $\left(a_{k}\right)_{n},\left(b_{k}\right)_{n},\left(c_{k}\right)_{n}$, and $\left(d_{k}\right)_{n}$ of $(1,-1)$ sequences is said to be a quad of Goethals-Seidel sequences of length $n$ (abbreviated as GSS $(n)$ ), if their associated polynomials satisfy

$$
|A(w)|^{2}+|B(w)|^{2}+|C(w)|^{2}+|D(w)|^{2}=4 n \quad \text { for any } w \in K_{n} .
$$

A sequence of vectors, $\left(v_{k}\right)_{n}$ is an $m$-symbol $\delta$-code of length $n$ if

$$
\sum_{k=1}^{n-j} v_{k} \cdot v_{k+j}=0 \quad \text { for each } j \neq 0
$$

where $v_{k}$ is one of $m$ orthonormal vectors $i_{1}, \ldots, i_{m}$, or their negatives (see [7]).

Definition. A quadruple $\left(q_{k}\right),\left(r_{k}\right),\left(s_{k}\right)$, and $\left(t_{k}\right)$ of $(0, \pm 1)$ sequences is said to be a quad of Turyn sequences (abbreviated as TS $(n)$ ) of length $n$, if the sequence $\left(v_{k}\right)_{n}$ of vectors $v_{k}=\left(q_{k}, r_{k}, s_{k}, t_{k}\right)$ forms a four-symbol $\delta$-code, where $v_{k}$ is one of orthonormal vectors $(1,0,0,0),(0,1,0,0),(0,0,1,0)$, and $(0,0,0,1)$, or their negatives.

Let $Q(z), R(z), S(z)$, and $T(z)$ be the associated polynomials of a given quad of $\operatorname{TS}(n),\left(q_{k}\right),\left(r_{k}\right),\left(s_{k}\right)$, and $\left(t_{k}\right)$. Then we have

$$
|Q(z)|^{2}+|R(z)|^{2}+|S(z)|^{2}+|T(z)|^{2}=n \quad \text { for any } z \in K .
$$

When $\left(a_{k}\right),\left(b_{k}\right),\left(c_{k}\right)$, and $\left(d_{k}\right)$ are a quad of $\operatorname{GSS}(n)$, they can be regarded, respectively, as the first row entries of $n \times n$ circulant matrices $A, B, C$, and $D$ such that $A A^{\prime}+B B^{\prime}+C C^{\prime}+D D^{\prime}=4 n I$. Then a Goethals-Seidel (Hadamard) matrix $H=\left(H_{i j}\right), 1 \leqslant i, j \leqslant 4$, of order $4 n$ can be formed by the sixteen $n \times n$ matrices $H_{i j}$ 
such that the first, second, third, and fourth rows of $H$ are, respectively, $(A, B R, C R, D R)$, $\left(-B R, A,-D^{\prime} R, C^{\prime} R\right),\left(-C R, D^{\prime} R, A,-B^{\prime} R\right)$, and $\left(-D R,-C^{\prime} R, B^{\prime} R, A\right)$, where $R=\left(r_{i j}\right), 1 \leqslant i, j \leqslant n$, is the $n \times n$ symmetric matrix whose entries $r_{i j}=1$ for $i+j=$ $n+1$ and $r_{i j}=0$, otherwise. (See [1], [7].)

Definition. A quad of $\operatorname{GSS}(n),\left(w_{k}\right),\left(x_{k}\right),\left(y_{k}\right)$, and $\left(z_{k}\right)$ is said to be a quad of Williamson sequences (abbreviated as WS $(n)$ ), if each sequence is symmetric, i.e. $a_{j}=$ $a_{n+2-j}$ for each $j$ and each $\left(a_{k}\right)$ of GSS $(n)$, or equivalently $A\left(w^{-1}\right)=A(w)$ for each $w \in K_{n}$ and each associated polynomial $A(w)$ of $\operatorname{GSS}(n)$.

It is well known that when $\left(w_{k}\right),\left(x_{k}\right),\left(y_{k}\right)$, and $\left(z_{k}\right)$ are a quad of WS $(n)$, they can be regarded as the first row entries of $n \times n$ symmetric circulant matrices $W, X$, $Y$, and $Z$, respectively, such that $W^{2}+X^{2}+Y^{2}+Z^{2}=4 n I$. Then a $4 \times 4$ matrix $H$ is a Williamson (Hadamard) matrix of order $4 n$, where $(W, X, Y, Z),(-X, W,-Z, Y)$, $(-Y, Z, W,-X)$, and $(-Z,-Y, X, W)$ are, respectively, the first, second, third, and fourth row blocks of $H$. (See [14], [15] , [16] .)

The following three theorems (on Hadamard sequences) are derived from the known theorems (on Golay complementary sequences). (See [2] , [7], and [10], respectively, for Theorems 2, 3, and 4.)

THEOREM 2. Let $\left(a_{k}\right)$ and $\left(b_{k}\right)$ be a pair of $\mathrm{GCL}(m)$ and $\left(c_{k}\right),\left(d_{k}\right)$, a pair of $\mathrm{HL}(n)$. Then $\left(e_{k}\right)$ and $\left(f_{k}\right)$ is a pair of $\mathrm{HL}(2 m n)$, where

$$
\begin{aligned}
& e_{(2 j-2) m+k}=a_{k} c_{j}, \quad e_{(2 j-1) m+k}=b_{k} d_{j} \text { and } f_{(2 j-2) m+k}=-a_{k} d_{n+1-j}, \\
& f_{(2 j-1) m+k}=b_{k} c_{n+1-j} \quad \text { for } 1 \leqslant k \leqslant m \text { and } 1 \leqslant j \leqslant n .
\end{aligned}
$$

Proof. Since

$$
\begin{aligned}
E(w) & =\sum_{1}^{2 m n} e_{k} w^{k-1}=\sum_{1}^{n}\left(c_{j} w^{2(j-1) m} A(w)+d_{j} w^{(2 j-1) m} B(w)\right) \\
& =A(w) C\left(w^{2 m}\right)+B(w) D\left(w^{2 m}\right) w^{m}
\end{aligned}
$$

and

$$
F(w)=\left(-A(w) D\left(w^{-2 m}\right)+B(w) C\left(w^{-2 m}\right) w^{m}\right) w^{-2 m} \quad \text { for any } w \in K_{2 m n},
$$

consequently $w^{2 m} \in K_{n}$, we therefore obtain from (1) and (3),

$$
|E(w)|^{2}+|F(w)|^{2}=\left(|A(w)|^{2}+|B(w)|^{2}\right)\left(\left|C\left(w^{2 m}\right)\right|^{2}+\left|D\left(w^{2 m}\right)\right|^{2}\right)=4 m n .
$$

THEOREM 3. Let $\left(a_{k}\right),\left(b_{k}\right)$ be a pair of $\mathrm{GCL}(m)$ and $\left(c_{k}\right),\left(d_{k}\right)$ be a pair of $\mathrm{HL}(n)$. Then $\left(e_{k}\right),\left(f_{k}\right)$ is a pair of $\mathrm{HL}(m n)$, where

$$
e_{(j-1) m+k}=\left[\left(a_{k}+b_{k}\right) c_{j}+\left(a_{k}-b_{k}\right) d_{j}\right] / 2
$$

and

$$
f_{(j-1) m+k}=\left[\left(a_{k}-b_{k}\right) c_{n+1-j}-\left(a_{k}+b_{k}\right) d_{n+1-j}\right] / 2
$$


Proof. Since

$$
E(w)=\left[(A(w)+B(w)) C\left(w^{m}\right)+(A(w)-B(w)) D\left(w^{m}\right)\right] / 2
$$

and

$$
\begin{aligned}
F(w)=\left[(A(w)-B(w)) C\left(w^{-m}\right)-(A(w)+B(w)) D\left(w^{-m}\right)\right] w^{-m} / 2 & \text { for any } w \in K_{m n},
\end{aligned}
$$

consequently $w^{m} \in K_{n}$, therefore, we have

$$
|E(w)|^{2}+|F(w)|^{2}=\left(|A(w)|^{2}+|B(w)|^{2}\right)\left(\left|C\left(w^{m}\right)\right|^{2}+\left|D\left(w^{m}\right)\right|^{2}\right) / 2=2 m n .
$$

It should be noted that if $\left(a_{k}\right)$ and $\left(b_{k}\right)$ are a pair of GCL $(m)$, then the sequence $\left(v_{k}\right)$ of vectors $v_{k}=\left(x_{k}, y_{k}\right)$, where $x_{k}=\left(a_{k}+b_{k}\right) / 2$ and $y_{k}=\left(a_{k}-b_{k}\right) / 2$, is a two-symbol $\delta$-code of length $m$ with the two orthonormal vectors $i_{1}=(1,0)$ and $i_{2}=(0,1)$, and conversely.

THEOREM 4. Let $\left(a_{k}\right)$ and $\left(b_{k}\right)$ be a pair of HL(n). Then $\left(a_{k}^{e}\right),\left(b_{k}^{e}\right) ;\left(a_{k}^{e}\right),\left(b_{k}^{0}\right)$; $\left(a_{k}^{0}\right),\left(b_{k}^{e}\right)$; and $\left(a_{k}^{0}\right),\left(b_{k}^{0}\right)$ are also pairs of $\mathrm{HL}(n)$, where $\left(c_{k}^{e}\right)$ is the sequence obtained from $\left(c_{k}\right)$ by changing the sign of $c_{k}$ if and only if the subscript $k$ is even, i.e. $c_{k}^{e}=$ $(-1)^{k-1} c_{k}$, and $c_{k}^{0}=(-1)^{k} c_{k}$ for $c_{k}=a_{k}$ or $b_{k}$.

Proof. Let $A(w)=A_{0}\left(w^{2}\right)+w A_{e}\left(w^{2}\right)$ and $B(w)=B_{0}\left(w^{2}\right)+w B_{e}\left(w^{2}\right)$ be, respectively, associated polynomials of $\left(a_{k}\right)$ and $\left(b_{k}\right)$. Then $A^{e}(w)=A_{0}\left(w^{2}\right)-w A_{e}\left(w^{2}\right)$, $B^{e}(w)=B_{0}\left(w^{2}\right)-w B_{e}\left(w^{2}\right), A^{0}(w)=-A_{0}\left(w^{2}\right)+w A_{e}\left(w^{2}\right)$, and $B^{0}(w)=-B_{0}\left(w^{2}\right)+$ $w B_{e}\left(w^{2}\right)$ are, respectively, associated polynomials of $\left(a_{k}^{e}\right),\left(b_{k}^{e}\right),\left(a_{k}^{0}\right)$, and $\left(b_{k}^{0}\right)$. Since $|A(w)|^{2}+|B(w)|^{2}=\left|A_{0}\left(w^{2}\right)+w A_{e}\left(w^{2}\right)\right|^{2}+\left|B_{0}\left(w^{2}\right)+w B_{e}\left(w^{2}\right)\right|^{2}=2 n$ for any $w \in K_{n}$, which is equivalent to $\left|A_{0}\left(w^{2}\right)\right|^{2}+\left|A_{e}\left(w^{2}\right)\right|^{2}+\left|B_{0}\left(w^{2}\right)\right|^{2}+\left|B_{e}\left(w^{2}\right)\right|^{2}$ $=2 n$ and

$$
\begin{aligned}
& w\left(A_{0}\left(w^{-2}\right) A_{e}\left(w^{2}\right)+B_{0}\left(w^{-2}\right) B_{e}\left(w^{2}\right)\right) \\
& \quad+w^{-1}\left(A_{0}\left(w^{2}\right) A_{e}\left(w^{-2}\right)+B_{0}\left(w^{2}\right) B_{e}\left(w^{-2}\right)\right)=0
\end{aligned}
$$

for any $w \in K_{n} \cdot{ }^{*}$ Consequently, we have

$$
\begin{aligned}
\left|A^{e}(w)\right|^{2}+\left|B^{e}(w)\right|^{2} & =\left|A_{0}\left(w^{2}\right)-w A_{e}\left(w^{2}\right)\right|^{2}+\left|B_{0}\left(w^{2}\right)-w B_{e}\left(w^{2}\right)\right|^{2} \\
& =\left|A_{0}\left(w^{2}\right)\right|^{2}+\left|A_{e}\left(w^{2}\right)\right|^{2}+\left|B_{0}\left(w^{2}\right)\right|^{2}+\left|B_{e}\left(w^{2}\right)\right|^{2}=2 n .
\end{aligned}
$$

Other cases can be proved similarly.

THEOREM 5. Let $\left(w_{k}\right),\left(x_{k}\right),\left(y_{k}\right)$, and $\left(z_{k}\right)$ be a quad of WS $(m)$ and $\left(q_{k}\right)$, $\left(r_{k}\right),\left(s_{k}\right)$, and $\left(t_{k}\right)$ a quad of $\mathrm{TS}(n)$. Then $\left(a_{k}\right),\left(b_{k}\right),\left(c_{k}\right)$, and $\left(d_{k}\right)$ are a quad of $\mathrm{GSS}(m n)$, where

$$
\begin{aligned}
& a_{(h-1) n+j}=w_{h} q_{j}+x_{h} r_{j}+y_{h} s_{j}+z_{h} t_{j}, \\
& b_{(h-1) n+j}=x_{h} q_{j}-w_{h} r_{j}+z_{h} s_{j}-y_{h} t_{j}, \\
& c_{(h-1) n+j}=y_{h} q_{j}-z_{h} r_{j}-w_{h} s_{j}+x_{h} t_{j}, \\
& d_{(h-1) n+j}=z_{h} q_{j}+y_{h} r_{j}-x_{h} s_{j}-w_{h} t_{j} \quad \text { for } 1 \leqslant h \leqslant m \text { and } 1 \leqslant j \leqslant n .
\end{aligned}
$$

${ }^{*}$ We use the fact that $w \in K_{n}$ implies $-w \in K_{n}$ for even $n$. 
Proof. For any $w \in K_{m n}$, we have

$$
\begin{aligned}
A(w) & =\sum_{1}^{m n} a_{k} w^{k-1}=\sum_{1}^{m} \sum_{1}^{n}\left(w_{h} q_{j}+x_{h} r_{j}+y_{h} s_{j}+z_{h} t_{j}\right) w^{(h-1) n+j-1} \\
& =W\left(w^{n}\right) Q(w)+X\left(w^{n}\right) R(w)+Y\left(w^{n}\right) S(w)+Z\left(w^{n}\right) T(w)
\end{aligned}
$$

similarly,

$$
\begin{aligned}
& B(w)=X\left(w^{n}\right) Q(w)-W\left(w^{n}\right) R(w)+Z\left(w^{n}\right) S(w)-Y\left(w^{n}\right) T(w) \\
& C(w)=Y\left(w^{n}\right) Q(w)-Z\left(w^{n}\right) R(w)-W\left(w^{n}\right) S(w)+X\left(w^{n}\right) T(w)
\end{aligned}
$$

and

$$
D(w)=Z\left(w^{n}\right) Q(w)+Y\left(w^{n}\right) R(w)-X\left(w^{n}\right) S(w)-W\left(w^{n}\right) T(w) .
$$

Since $w^{n} \in K_{m}$ and $U\left(w^{-n}\right)=U\left(w^{n}\right)$ for $U=W, X, Y$, and $Z$, by replacing the righthand sides of the above into the following sum and by rearrangements and simplifications, we obtain from (5) and (7),

$$
\begin{aligned}
& |A(w)|^{2}+|B(w)|^{2}+|C(w)|^{2}+|D(w)|^{2} \\
& =\left(W^{2}+X^{2}+Y^{2}+Z^{2}\right)\left(|Q|^{2}+|R|^{2}+|S|^{2}+|T|^{2}\right)=4 m n
\end{aligned}
$$

where $U=U\left(w^{n}\right)$ for $U=W, X, Y$, and $Z ; P=P(w)$ for $P=Q, R, S$, and $T$.

It should be noted that a Hadamard matrix of order $4 m n$ has been constructed by Turyn [7] using Baumert-Hall units if a Williamson matrix of order $4 m$ and a foursymbol $\delta$-code of length $n$ are known. Williamson matrices of order $4 m$ exist for $m \leqslant 31$ or $m=(q+1) / 2$, where $q$ (a prime power) $\equiv 1(\bmod 4)$, and others (see [4], [7], [8], [11], [13], [14], [15]).

Four-symbol $\delta$-codes (including two- and three-symbol codes) of length $n$ exist for $n \leqslant 61$, or $n=2^{a} 10^{b} 26^{c}$ (two-symbol codes) and $n=2^{a} 10^{b} 26^{c}+1$ (three-symbol codes) for all $a, b, c \geqslant 0$ (see [7], [9], [11]), as well as for $n=2^{a} 10^{b} 26^{c}+2^{d} 10^{e} 26^{f}$ (four-symbol codes) for all $a, b, c, d, e$, and $f \geqslant 0$.

For example, in the two-symbol $\delta$-code $(1, i)$ of length 2 , by letting $1=$ $(1,0,0,0)$ and $i=(0,1,0,0)$, we obtain a quad of $\operatorname{TS}(2):\left(q_{k}\right)=(1,0),\left(r_{k}\right)=$ $(0,1)$, and $\left(s_{k}\right)=\left(t_{k}\right)=(0,0)$. Similarly, by letting 1 and $i$ as above and $j=$ $(0,0,1,0)$ in the three-symbol code $(1, i, j)$ of length 3 , we obtain a quad of TS(3): $\left(q_{k}\right)=(1,0,0),\left(r_{k}\right)=(0,1,0),\left(s_{k}\right)=(0,0,1)$, and $\left(t_{k}\right)=(0,0,0)$. Thus, for the given quad of WS(3): $\left(w_{k}\right)=\left(x_{k}\right)=\left(y_{k}\right)=(-, 1,1)$ and $\left(z_{k}\right)=(1,1,1)$, where stands for -1 , we obtain from Theorem 5 the following quads of $\operatorname{GSS}(n)$ for $n=6$ and 9. $n=6:\left(a_{k}\right),\left(b_{k}\right),\left(c_{k}\right)$, and $\left(d_{k}\right)$ are, respectively, $(-,-, 1,1,1,1),(-, 1,1$, $-, 1,-),(-,-, 1,-, 1,-)$, and $(1,-, 1,1,1,1) ; n=9:(-,-,-, 1,1,1,1,1,1)$, $(-, 1,1,1,-, 1,1,-, 1),(-,-, 1,1,-,-, 1,-,-)$, and $(1,-, 1,1,1,-, 1,1,-)$.

The following new pairs $\left(a_{k}\right)$ and $\left(b_{k}\right)$ of $\operatorname{HL}(26)$ have been found, which are listed as the following pairs of $C$ and $D$, respectively, where $C=\left\{k: a_{k}=-1\right\}$ and $D=\left\{k: b_{k}=-1\right\} . C$ and $D$ are: $\{1,2,3,7,9,11,12,14,15,17,18\}$ and $\{1,2$, $4,8,9,11,13,16,17,21\} ;\{1,2,3,5,6,8,12,13,15,21\}$ and $\{1,2,3,5,7,11$, $12,13,16,19,24\} ;\{1,2,4,6,8,9,12,13,15,22\}$ and $\{1,2,3,4,8,9,13,16$, $17,19,25\}$; and $\{1,2,3,5,6,10,12,13,15,23\}$ and $\{1,2,3,7,9,10,12,13,16$, 
$21,23\}$, respectively. From Theorem 4, we also obtain the following pair corresponding to $\left(a_{k}^{0}\right)$ and $\left(b_{k}^{0}\right)$ of the first pair above, $\{2,5,12,13,14,18,19,21,23,25\}$ and $\{2,3,4,5,7,8,15,16,19,23,25\}$. Other pairs of $\mathrm{HL}(26)$ corresponding to $\left(a_{k}^{0}\right)$ and $\left(b_{k}^{0}\right)$, or $\left(a_{k}^{e}\right)$ and $\left(b_{k}^{e}\right)$, can be obtained from Theorem 4 in a similar way. We note here that $\left(c_{k}^{e}\right)=\left(-c_{k}^{0}\right)$ for $c_{k}=a_{k}$ or $b_{k}$.

In Theorem 3, for example, from the given pairs of GCL(10) and HL(4): $\left(a_{k}\right)=$ $(1,1,1,1,-, 1,1,-,-, 1),\left(b_{k}\right)=(1,-, 1,-, 1,1,1,1,-,-)$ and $\left(c_{k}\right)=\left(d_{k}\right)=$ $(-, 1,1,1)$, we obtain the following $E$ and $F$ representing the pair $\left(e_{k}\right)$ and $\left(f_{k}\right)$ of HL(40):

$$
E=\left\{k: e_{k}=-1\right\}=\{1,2,3,4,6,7,10,15,18,19,25,28,29,35,38,39\}
$$

and

$$
\begin{aligned}
F & =\left\{k: f_{k}=-1\right\} \\
& =\{1,3,5,6,7,8,11,13,15,16,17,18,21,23,25,26,27,28,32,34,39,40\} .
\end{aligned}
$$

Department of Mathematical Sciences

State University of New York, College at Oneonta

Oneonta, New York 13820

1. J. M. GOETHALS \& J. J. SEIDEL, “A skew Hadamard matrix of order 36," J. Austral. Math. Soc., v. 11, 1970, pp. 343-344.

2. M. J. E. GOLAY, "Complementary series," IRE Trans. Information Theory, v. IT-7, 1961 , pp. 82-87.

3. M. J. E. GOLAY, "Note on complementary series," Proc. IRE, v. 50, 1962, p. 84.

4. E. SPENCE, "Hadamard matrices of order $2 q^{r}(q+1)$ and $q^{r}(q+1)$," Notices Amer. Math. Soc., v. 23, 1976, p. A-353.

5. E. SPENCE, "Hadamard matrices of the Goethals-Seidel type," Canad. J. Math., v. 27, 1975 , pp. 555-560.

6. E. SPENCE, "Skew-Hadamard matrices of order $2(q+1)$," Notices Amer. Math. Soc., v. 22, 1975, p. A-303.

7. R. J. TURYN, "Hadamard matrices, Baumert-Hall units, four-symbol sequences, pulse compression, and surface wave encodings," J. Combinatorial Theory Ser. A, v. 16, 1974, pp. 313333.

8. R. J. TURYN, “An infinite class of Williamson matrices," J. Combinatorial Theory Ser. $A$, v. 12, 1972, pp. 319-321.

9. R. J. TURYN, “Computation of certain Hadamard matrices," Notices Amer. Math. Soc., v. 20, 1973, p. A-1.

10. Y. TAKI et al., "Even-shift orthogonal sequences," IEEE Trans. Information Theory, v. IT-15, 1969, pp. 295-300.

11. J. S. WALLIS, “On Hadamard matrices," J. Combinatorial Theory Ser. A, v. 18, 1975, pp. $149-164$.

12. A. L. WHITEMAN, "Skew Hadamard matrices of Goethals-Seidel type," Discrete Math., v. 2, 1972, pp. 397-405.

13. A. L. WHITEMAN, "Williamson type matrices of order $2 q(q+1)$," Notices Amer. Math. Soc., v. 21, 1974, p. A-623.

14. A. L. WHITEMAN, "An infinite family of Hadamard matrices of Williamson type," $J$. Combinatorial Theory Ser. A, v. 14, 1973, pp. 334-340.

15. J. WILLIAMSON, "Hadamard's determinant theorem and sum of four squares," Duke Math. J., v. 11, 1944, pp. 65-81.

16. C. H. YANG, "On Hadamard matrices constructible by circulant submatrices," Math. Comp., v. 25, 1971, pp. 181-186.

17. C. H. YANG, "Maximal binary matrices and sum of two squares," Math. Comp., v. 30, 1976, pp. $148-153$. 